\title{
Massive choleperitoneum three months after mini-gastric bypass for morbid obesity: what every emergency surgeon should be prepared to face. A case report.
}

Giacomo Piatto $^{1 *}$, Alberto Sartori ${ }^{1}$, Maurizio De Luca ${ }^{1}$

${ }^{1}$ UOC Chirurgia Generale, Ospedale di Montebelluna (TV), Italia

To Cite

Piatto G, Sartori A, De Luca M. Massive choleperitoneum three months after minigastric bypass for morbid obesity: what every emergency surgeon should be prepared to face. A case report. J Gastric Surg 2020; 2(2): 49-52

\section{Publication history}

Received: March 26, 2020

Accepted: March 28, 2020

Article in press: March 31, 2020

Published on line: April 1, 2020

\section{*Correspondence to}

Dr. Giacomo Piatto

UOC Chirurgia Generale,

Ospedale di Montebelluna,

31044 Montebelluna (TV), Italy

giacomoplatz@gmail.com

Telephone: +393407931459

\begin{abstract}
Background:

Surgery for morbid obesity has spread worldwide, to the point that more than half a million people are operated on each year. As a result, significant numbers of people are living with a new anatomical condition. A mini-gastric bypass is a relatively new bariatric procedure that has gained popularity because of its simplicity and efficacy. Leak rate after this procedure is relatively low (on the order of $1.6 \%$ ), but marginal ulcer of gastrojejunal anastomosis, if undetected, may lead to leak development.

No cases of delayed massive choleperitoneum caused by an almost complete disruption of gastrojejunal anastomosis after mini-gastric bypass have yet been described.

\section{Case presentation:}

We describe here the case of a 51-year-old woman who presented at the emergency department three months after a mini-gastric bypass with acute abdomen caused by massive choleperitoneum due to an almost complete disruption of gastrojejunal anastomosis.

The patient underwent an emergency conversion to a Roux-en-Y laparoscopic gastric bypass with associated re-gastrectomy. The postoperative period was characterized by fever due to an infected left pleural effusion, which required treatment with chest tube placement. The patient was discharged three weeks after the operation, in good condition. Six-month follow-up was regular.
\end{abstract}

\section{Conclusions:}

If suspected, the possibility of marginal ulcer should be investigated as soon as possible. When possible, every obese patient who has complications should be referred to a bariatric surgery department, but each emergency surgeon must be aware of these conditions to be able to treat them optimally.

Keywords:

Mini-gastric bypass, complication, leak, marginal ulcer, emergency surgery, gastrectomy, laparoscopy, choleperitoneum. 


\section{Background:}

Mini-gastric bypass (MGB) was proposed by Rutledge in 1996[1]. This technique overcame initial suspicion to gain worldwide popularity for its effectiveness and simplicity and is now considered a valid option for bariatric operation[2].

The technique involves creating a long, narrow gastric pouch by transecting the stomach below the crow's foot up to the angle of His, then attaching the pouch through an end-to-side single anastomosis with the jejunum, 200-250 cm from Treitz.

Leak rate after MGB is relatively low (O.8-1.6\%), especially compared with the rates of other malabsorptive procedures such as Roux-en-Y gastric bypass (RYGB), which can reach $2.5 \%$ [3].

Leak usually develops within the first month after the operation; as reported in various studies, septic patients present symptoms within the first 10 days after MGB[4]. No cases of delayed massive choleperitoneum caused by almost complete disruption of the gastrojejunal anastomosis after this operation have thus far been described in the literature.

Bariatric procedures have gained worldwide popularity, with more than half a million performed each year[5]. However, patients may present to the emergency department, where no bariatric surgeons may be available or even on call. Although these patients should be referred to their bariatric surgery department when possible, in emergency scenarios this is not always possible.

\section{Case Report:}

A 51-year-old female patient underwent sleeve gastrectomy in March 2018 for morbid obesity (weight $106 \mathrm{~kg}$, BMI 40). In May 2018 she required conversion to MGB for severe stricture of the sleeved stomach. During the initial follow-up, she was taking a full dose of lansoprazole ( $30 \mathrm{mg}$ twice a day) and was still on a soft diet.

In June, at first-month follow-up, the patient suffered from severe intermittent abdominal pain in the left hypochondrium and epigastrium. A CT scan was performed, with an internal hernia suspected. The patient was admitted to our department and a diagnostic laparoscopy was performed. No signs of internal hernia or any pathological conditions inside the abdominal cavity were detected. The patient was prescribed additional sucralfate and was discharged the day after the operation. No endoscopy was performed during the next two months, for the patient did not complain of any symptoms.

In August 2018 the patient returned to our emergency department complaining of acute abdominal pain and fever, with reported oliguria. The patient presented with tachycardia (heart rate reaching $110 \mathrm{bpm}$ ), hypotension (blood pressure reaching 90/60 $\mathrm{mmHg}$ ), fever, and abdominal rigidity, with diffuse signs of peritoneal irritation; blood tests showed significant leukocytosis (WBCs count of $24000 / \mathrm{mcL}$ ) and raised CRP levels $(13 \mathrm{mg} / \mathrm{L})$. The patient immediately underwent a CT scan showing free gas and free fluid spread within the abdominal cavity (Figure 1).

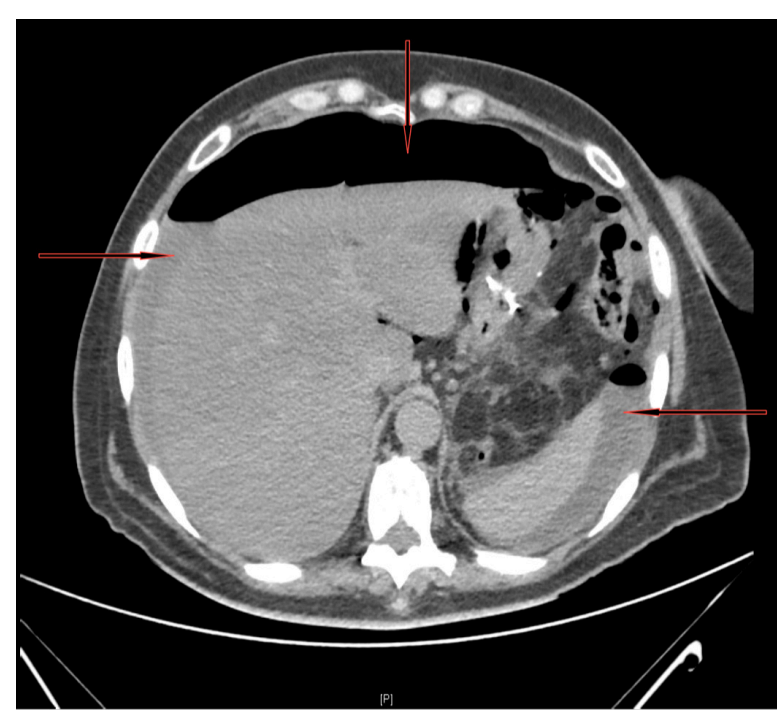

Figure 1: CT scan showing intra-abdominal free fluid and free gas (arrows).

The patient was immediately brought to the operating room, where a diagnostic laparoscopy was performed. The patient was positioned open-legged, with the first operator between the legs and the assistant to the left of the patient, holding the camera and using a $5-\mathrm{mm}$ port. We induced the pneumoperitoneum with a Veress needle in the left Palmer point, then introduced a 30-degree camera through a 12-mm Visiport over the umbilicus and found a massive choleperitoneum (Figure 2).
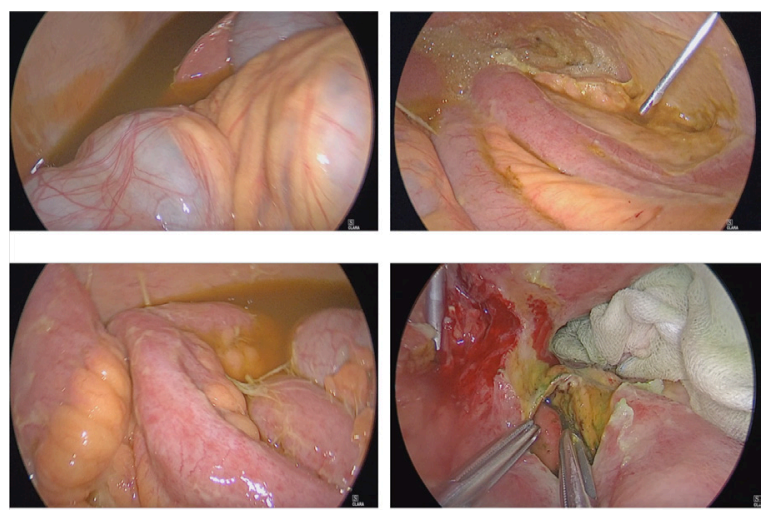

Figure 2: Massive choleperitoneum as the result of almost complete disruption of the gastrojejunal anastomosis.

There were no significant adhesions inside the abdominal cavity. Two operative $12-\mathrm{mm}$ trocars were introduced into the left and right flank of the patient, and another 5 -mm trocar was inserted more laterally into the left flank. The left lobe of the liver was suspended through a covered Veress needle introduced just below the xiphoid, anchored to the skin with a stitch.

After the aspiration of more than two liters of bilioenteric fluid, a thorough exploration of the bowel was carried out. Finding almost complete disruption of the gastrojejunal anastomosis (Figure 2), we decided to completely resect the anastomosis, conducting a regastrectomy and resection of both sides of the jejunum 
(common and biliopancreatic limb).

Reconstruction was immediately performed through a standard laparoscopic RYGB, with a manual end-to-end gastrojejunal anastomosis and a mechanical side-to-side jejunoileal anastomosis (Figure 3).
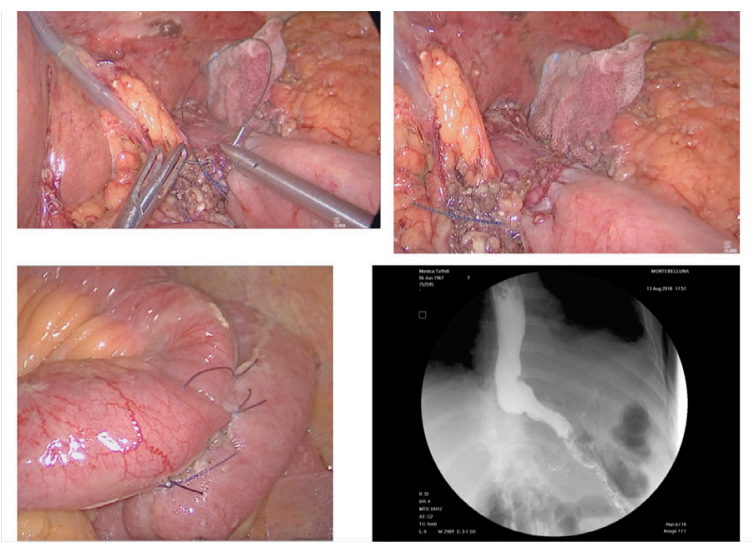

Figure 3: Emergency surgery. Laparoscopic MGB converted to laparoscopic RYGB with re-gastrectomy.

The methylene blue dye test was negative. The patient required 24-hour monitoring in the intensive care unit. She was then readmitted to our department and received total parenteral nutrition for five days.

Swallow X-ray examination on the second postoperative day was regular, and the patient began a soft diet at postoperative day 6 . At postoperative day 7 she required placement of a chest tube due to an infected left pleural effusion, which was successfully resolved after three days.

The patient was discharged three weeks after the operation and at six-month follow-up had no symptoms and had reached a weight of $75 \mathrm{~kg}$ and a BMI of 28.6. Histopathological examination showed full-thickness ulceration and necrosis of the resected jejunum, also involving the anastomosis.

\section{Discussion:}

Leak rate after MGB, which is between $0.8 \%$ and $1.6 \%$, can be explained by the specific features of MGB: the long narrow gastric pouch created does not suffer from inner pressure, and the gastrojejunal anastomosis is tension-free[6]. Even so, more than half of leaks develop from the single anastomosis, with the remainder arising from the gastric remnant and the pouch itself.

Although some authors regard bile reflux as a factor related to leak development[7], others show that no specific factors are related to the onset of a fistula[8]. Leak may develop from an undetected marginal ulcer, a condition related to smoking, use of nonsteroidal antiinflammatory drugs, alcohol consumption, or presence of Helicobacter pylori[9].

A number of aspects must be taken into account when analyzing this problem: the patient's clinical condition, the leak's site and size, and the resources available (i.e., endoscopist, interventional radiologist, and bariatric surgeon).

In a 2017 survey Mahawar et al. analyzed more than 27,000 one-anastomosis (mini) gastric bypasses performed by 86 bariatric surgeons, seeking to understand the causes of marginal ulcer[9], a condition that in some cases leads to leakage of the single anastomosis. They found a $2.24 \%$ incidence of marginal ulcer, but only a few cases required surgery for perforation. They found a lack of standardization for prevention, diagnosis, and treatment of this complication.

Smoking, the use of nonsteroidal anti-inflammatory drugs, alcohol consumption, and Helicobacter pylori infection are the most important risk factors for marginal ulcers. None of these risk factors was present in our patient. In the cited survey by Mahawar, when marginal ulcer is suspected, endoscopy is routinely performed by only $58 \%$ of surgeons. We did not perform endoscopy in this case, because the patient responded well to a full dose of proton pump inhibitor added with sucralfate. In a recent series of 2,780 patients who underwent one-anastomosis gastric bypasses, the authors tried to identify the best way of treating leaks after a MGB in relation to the clinical conditions of patients (stable vs. unstable) and the size and site of the leak, finding that with a leak rate of $1.6 \%$ (46 patients), only $28 \%$ required surgical exploration.

Of the five septic patients, only one required conversion to RYGB for early gastrojejunal anastomotic leak; the others were treated with laparoscopic T-tube placement. All five septic patients presented at the hospital within 10 days of the operation, much sooner than in our case. Revisional MGB was shown to be associated with a higher risk of staple line leak in the gastrojejunal anastomosis than with the primary operation[4]. Similar results in terms of leak rate and surgical approach were described in a previous survey[10]. The literature (Pubmed, Medline, Google Scholar) showed no results for delayed massive choleperitoneum after MGB, leading the authors of the present study to expand the research into the field of emergency surgery. Laparoscopy in emergency surgery, which has gained increasing popularity, represents the gold standard for treatment of appendicitis and cholecystitis and is a useful tool even in trauma, as described in a recent review [11]. Furthermore, its superiority to laparotomy in perforated peptic ulcer repair has been shown in a recent meta-analysis[12].

Merging all these considerations, we conclude that situations such as the one we encountered must be treated aggressively by emergency surgeons and can be successfully managed by using a laparoscopic approach both to identify the site of the perforation and to treat it.

\section{Conclusion:}

Through the present study we hope to raise awareness among emergency surgeons so that they can treat dramatic complications such as the one we encountered. The suspected etiology may reflect the combined effects of revisional surgery performed in a patient suffering from GERD and an undetected marginal ulcer.

After three months of bariatric surgery, the likelihood of a complicated septic patient's reaching an emergency department with no bariatric surgeons present or on call is a real one. Accordingly, every emergency surgeon must be aware of the anatomical changes that take place after bariatric procedures if he or she is to address potentially lethal complications in the quickest and most suitable way. 


\section{Acknowledgements}

Not applicable.

\section{Contributors}

Each of the listed authors made a substantial contribution to the conception, design, and revision of this study. All authors read and approved the final manuscript.

\section{Funding}

No funding was received for this study.

\section{Competing interests}

No benefits in any form have been received or will be received from a commercial party related directly or indirectly to the subject of this article.

\section{Availability of data and materials}

Further information is available from the corresponding author on reasonable request.

\section{Ethics approval}

written informed consent was obtained from the patient for publication of this case report and any accompanying images.

\section{Provenance and peer review}

Not commissioned; externally peer reviewed.

\section{Open access}

This is an Open Access article distributed in accordance with the Creative Commons Attribution NonCommercial (CC BY-NC 4.0) license, which permits others to distribute, remix, adapt, build upon this work noncommercially, and license their derivative works on different terms, provided the original work is properly cited and the use is non-commercial. See: http://creativecommons.org/ licenses/by-nc/4.0/

\section{References}

[1] Rutledge R. The mini-gastric bypass: experience with the first 1,274 cases. Obes Surg. 2001;11:276-80.

[2] De Luca M, Tie T, Ooi G, Higa K, Himpens J, Carbajo MA, et al. Mini Gastric Bypass-One Anastomosis Gastric Bypass (MGB-OAGB)IFSO Position Statement. Obes Surg. 2018;28:1188-206.

[3] Kim J, Azagury D, Eisenberg D, DeMaria E, Campos GM, American Society for M, et al. ASMBS position statement on prevention, detection, and treatment of gastrointestinal leak after gastric bypass and sleeve gastrectomy, including the roles of imaging, surgical exploration, and nonoperative management. Surg Obes Relat Dis. 2015;11:739-48.

[4] Liagre A, Queralto M, Juglard G, Anduze Y, Iannelli A, Martini F. Multidisciplinary Management of Leaks After One-Anastomosis Gastric Bypass in a Single-Center Series of 2780 Consecutive Patients. Obes Surg. 2019;29:1452-61.

[5] Angrisani L, Santonicola A, Iovino P, Vitiello A, Zundel N, Buchwald H, et al. Bariatric Surgery and Endoluminal Procedures: IFSO Worldwide Survey 2014. Obes Surg. 2017;27:2279-89.

[6] Rutledge R, Kular K, Manchanda N. The Mini-Gastric Bypass original technique. Int J Surg. 2019;61:38-41.

[7] Mahawar KK, Jennings N, Brown J, Gupta A, Balupuri S, Small PK. "Mini" gastric bypass: systematic review of a controversial procedure. Obes Surg. 2013;23:1890-8.

[8] Silecchia G, Iossa A. Complications of staple line and anastomoses following laparoscopic bariatric surgery. Ann Gastroenterol. 2018;31:56-64.

[9] Mahawar KK, Reed AN, Graham YNH. Marginal ulcers after one anastomosis (mini) gastric bypass: a survey of surgeons. Clin Obes. 2017;7:151-6.

[10] Musella M, Susa A, Manno E, De Luca M, Greco F, Raffaelli M, et al. Complications Following the Mini/One Anastomosis Gastric Bypass (MGB/OAGB): a Multi-institutional Survey on 2678 Patients with a Mid-term (5 Years) Follow-up. Obes Surg. 2017;27:2956-67.

[11] Mandrioli M, Inaba K, Piccinini A, Biscardi A, Sartelli M, Agresta $\mathrm{F}$, et al. Advances in laparoscopy for acute care surgery and trauma. World J Gastroenterol. 2016;22:668-80.

[12] Tan S, Wu G, Zhuang Q, Xi Q, Meng Q, Jiang Y, et al. Laparoscopic versus open repair for perforated peptic ulcer: A meta analysis of randomized controlled trials. Int J Surg. 2016;33 Pt A:124-32. 